\title{
IPR tracking system in Collaborative Environments
}

\author{
G. Fantoni ${ }^{1}$, R. Apreda ${ }^{1}$, P. Valleri ${ }^{1}$, A. Bonaccorsi ${ }^{2}$, M. Manenti ${ }^{3}$ \\ ${ }^{1}$ Department of Mechanical, Nuclear and Production Engineering, University of Pisa, Italy, \\ g.fantoni@ing.unipi.it,apreda@df.unipi.it,ilvalle@email.it \\ ${ }^{2}$ Department of Electrical System, University of Pisa, Italy, a.bonaccorsi@gmail.com \\ ${ }^{3}$ Department of Private Legal Studies, University of Siena, Italy, micolmanenti@libero.it
}

\begin{abstract}
The present paper illustrates a novel model for collaborative crowdsourcing and other collaborative environments where IPR tracking and protection constitutes a key issue. After a comparison between different approaches for innovation and R\&D, the new architecture is introduced, with a focus on problem solving activities. The study also investigates the complex IPR framework necessary to involve firms and to promote users' participation exploiting simultaneously collaboration and meritocracy. The paper also presents an original software application tool for tracing and tracking the IPR generated in collaborative and Open Innovation environments. The software's use and results are demonstrated through a case study.
\end{abstract}

\section{Keywords}

Open Innovation, Collaborative Environments, IPR tracking, IPR management, Problem Solving

\section{Introduction and state of the art}

In response to the more and more dynamical market conditions, companies tend to externalize many activities, including product design and R\&D which are substituted with outsourcing or crowdsourcing.

In the outsourcing, firms form temporary groups of employers and consultants who work together to solve a problem or design a new product.

In the crowdsourcing the solution of a problem is outsourced to a crowd of individuals via web. Simultaneously they reduce the R\&D team to a small group of experts able to validate the solutions coming from the crowd.

Both systems are based upon the principles of Open Innovation, but they do not exploit all the potentialities of diversity and collaboration. In the last ten years several examples of Open Innovation with a more collaborative philosophy spread out from Open source software to living labs etc.. but sometimes those approaches struggle to become economically viable.

Therefore the challenge is to combine multidisciplinarity, collaboration and meritocracy. Collaboration of many people shortens development times; multidisciplinarity allows the team to tackle problems from different points of view; meritocracy motivates the participant and fosters the free exchange of ideas. Such a synergy could translate into better solutions obtained in less time [Pisano 2004; Bifulco 2005].

The main benefits and disadvantages of different approaches to problem solving are summarized in Table 1 [Fleming 2004, Fantoni 2006]. 


\begin{tabular}{|c|c|c|}
\hline Type & Advantages & Drawbacks \\
\hline $\begin{array}{l}\text { Standard } \\
\text { approach }\end{array}$ & $\begin{array}{l}\text { - Know-how kept in the } \\
\text { company, } \\
\text { - Employees are well rewarded } \\
\text { and loyal to firms }\end{array}$ & $\begin{array}{l}\text { - High cost for the firm, } \\
\text { - Low efficiency compared to } \\
\text { other approaches, } \\
\text { - Difficult to achieve } \\
\text { breakthrough ideas }\end{array}$ \\
\hline Outsourcing & $\begin{array}{l}\text { - Cost reduction, } \\
\text { - Access to new competencies } \\
\text { and expertises }\end{array}$ & $\begin{array}{l}\text { - Know-how partially } \\
\text { outsourced, } \\
\text { - Dissatisfaction of external } \\
\text { workers, } \\
\text { - "Not Invented Here" syndrome }\end{array}$ \\
\hline Crowdsourcing & $\begin{array}{l}\text { - Enormous cost reduction, } \\
\text { - Pay only after the solution is } \\
\text { achieved, } \\
\text { - Maximum access to new } \\
\text { external expertises }\end{array}$ & $\begin{array}{l}\text { - Loss of many internal expert } \\
\text { resources; } \\
\text { - One wins, all the others lose; } \\
\text { - The generated knowledge is not } \\
\text { shared }\end{array}$ \\
\hline Open Source & $\begin{array}{l}\text { - Collaborative environment, } \\
\text { - IPR is tracked and managed }\end{array}$ & $\begin{array}{l}\text { - Voluntary contribution, } \\
\text { - Many methods can not be } \\
\text { extended outside software }\end{array}$ \\
\hline Open Innovation & $\begin{array}{ll}\text { - } & \text { Collaborative environment } \\
\text { - } & \text { User centred innovation }\end{array}$ & $\begin{array}{l}\text { - Voluntary contribution, not } \\
\text { business oriented } \\
\text { - Difficulties in fair revenues } \\
\text { sharing }\end{array}$ \\
\hline $\begin{array}{l}\text { Collaborative } \\
\text { Crowdsourcing }\end{array}$ & $\begin{array}{l}\text { - } \text { Pay per solution, } \\
\text { - } \quad \text { Collaborative environment, } \\
\text { - High effectiveness and } \\
\text { efficiency }\end{array}$ & $\begin{array}{l}\text { - Management of collaboration } \\
\text { and contracts, } \\
\text { - IPR issues and revenues } \\
\text { sharing not managed in a } \\
\text { systematic way }\end{array}$ \\
\hline
\end{tabular}

Table 1: Advantages and drawbacks of different approaches

The new paradigm of collaborative crowdsourcing is starting to appear in the web. For example, http://www.kluster.com and http://www.namethis.com successfully solve problems or provide services by exploiting the strength of collaboration. The main problem of such experiences is the absence of an IPR tracking system, as stressed by their own users.

Our paper proposes a possible approach to collaborative crowdsourcing and related IPR issues. Since IPR issues are strictly connected with knowledge, social, business [Bifulco 2005] and legal aspects a preamble on the reference environment and on the main consequences at legal and users level is necessary and is presented hereinafter in the following subsessions.

\subsection{The reference environment}

To overcome the dichotomy between meritocracy and collaboration we propose a new model of collaborative crowdsourcing. The model has been developed, both at theoretical and practical level, by the research group of the Leaning Lab (the Living Lab established in Pisa, Italy).

Among the numerous activities of the Leaning Lab a particular role is played by problem solving on demand. With time, solvers have realized the usefulness and indeed the necessity of an appropriate way of tracking each participant's contribution and of sharing the profits accordingly. 
Therefore a reliable IPR tracking and protection protocol has been developed, tested and implemented in a dedicated software platform. Thanks to such tool, solvers can give their contributions confident in a fair reward.

\subsection{The users/participants perspective}

Even assuming that all algorithms and procedures are well founded on a logical/mathematical basis, two further aspects are crucial for the whole architecture to work in real life contexts.

The first is that users/participants need to perceive the system as objective. Hence the procedure should be as human-independent as possible. In the ideal situation all steps, from contributions' recording to revenues' redistribution, are automatically managed by a software. This way the objectivity of the result is guaranteed.

The second point is that the users need to perceive the system as transparent: i.e. parameters, weights and protocol must be public. The participants should be free to question and discuss the rules and the parameters before joining the session. Once a common view is achieved, solvers and enterprises alike will sign the agreed protocol and bind themselves to accept the results of the IPR tracking and revenues redistribution algorithms.

\subsection{Legal aspects}

Despite the proven effectiveness of collaborative innovation, many legal issues must be considered and can sometimes constitute an obstacle. Actually in collaborative environments, many different forms of contract have to be managed, different contracts can embed different IPR conditions, issues as Unfair Competition, Privacy and Confidentiality, Company Loyalty have to be carefully addressed when an internal problematic is disclosed to people external to the company. Indeed the management of Intellectual Property Rights changes from country to country and from company to company, making difficult to correctly address the issue in complex and heterogeneous environments such as collaborative and Open Innovation ones. See for more details ALIVE.

The various partners (companies, professionals, employees) involved may have different legal status (private enterprises, universities, public-owned research centres, professional men...) and different obligations (company's employees vs. temporary workers, public vs. private and so on). The intermediation between the commissioning enterprise and the research group is usually carried out by a third subject. In our case the mediator is the Leaning Lab, which also acts as provider of the IPR tracking tool.

Since the existence of such problems, before every session each participant, regardless of its status, must subscribe a non disclosure agreement. With such agreement the parties commit themselves to not reveal neither the content of the meeting(s), nor the information the enterprise had to provide in order to solve the assigned task. Participants who are someone else's employees must also declare the compatibility of the task at hand with the regulations concerning the loyalty to their employer.

If the solving activity has been commissioned, the commissioning enterprise keeps all the economic rights concerning the invention's exploitation, including the full liberty to patent it or not. On the contrary, the moral rights and the invention paternity are inalienable rights of the inventor, regardless of his economical or juridical relationship with the enterprise.

\section{Research Approach}

\subsection{General considerations on IPR tracking}

In standard crowdsourcing (only one winner) there is no need for measuring similarities/difference among the solutions. On the contrary, if we want to integrate the meritocratic and competitive approach of crowdsourcing with the fair collaborative 
environments typical of OS/OI environments, the assessment of each contribution become a key issue. To encourage participation, knowledge sharing and trustfulness it is necessary to build an environment (real or virtual) whose first rule is the fair distribution of rewards based on each participant's contribution. There is a compelling need for a unanimously recognized, meritocratic and fair system or protocol to distribute revenues.

Just keeping track of the solvers contributions, via recording voices, tracing emails or keeping the log of textual discussions via web, is not enough. Actually comparing the contributions and measuring the value of each contribution in an objective way is more complex goal.

One hint about possible paradigms comes from the Open Source world. Indeed, in the development of Open Source software, CV systems track the differences among following versions of the same code. Anyway developers do not considered them good tools for measuring the contributions, but are more interested in comparing and benchmarking the speed of a new algorithm.

Since problem solving sessions concerns technical problems, the measurements could be related to the functional features of the proposed solutions. Among the possible solutions (e.g. by creating abstract functional representations based on rigid design representation [Dong 1997] or on complex ontological frameworks [Kitamura 2001, Borgo 2008]) we decided to consider the semantic content [Cascini 2004] of the contributions, and use computational linguistic [Cascini and Neri 2004] inspired techniques to extract information and make comparisons.

When a suitable method for measuring differences and similarities between statements or ideas as concepts has been elaborated, the following step is the creation of an algorithm that:

1. individuates the key ideas or crucial points of the final solution;

2. for each of such key elements, analyses the brainstorming log in order to find the statement that originated it;

3. Combine all the relevant information in an aggregate data about each participant's contribution to the overall final solution.

Finally, another protocol will weight each contribution according to its relevance and divide the reward accordingly.

\subsection{The IPR tracking system}

The system we adopted at the Leaning Lab to collect all the contributions is a web forum: the post content, the date and time, and the name of the person are stored as they appear in the log of the forum.

The software platform that implements the IPR tracking system is composed of the mentioned web forum, a parser, a suitable knowledge Data Base (developed by the $L L$ research group with a conceptual and practical effort of over two years) and a web engine.

As shown in Figure 1, the parser parses the text, analyses the content of all the posts and disambiguates (automatically if possible or manually if not) those words (lemmas) with multiple meanings. Afterwards, semantic rules are applied to recognize synonyms, antonyms, iperonyms, etc. and the text is modified including additional information coming from the knowledge base. All posts are then indexed by a web engine. Finally the web engine searches the content of the post $\mathrm{i}$ on the other posts. Applying a series of formal rules (again developed by the $L L$ group) the web engine also measures the ranking among the $n$ posts. 


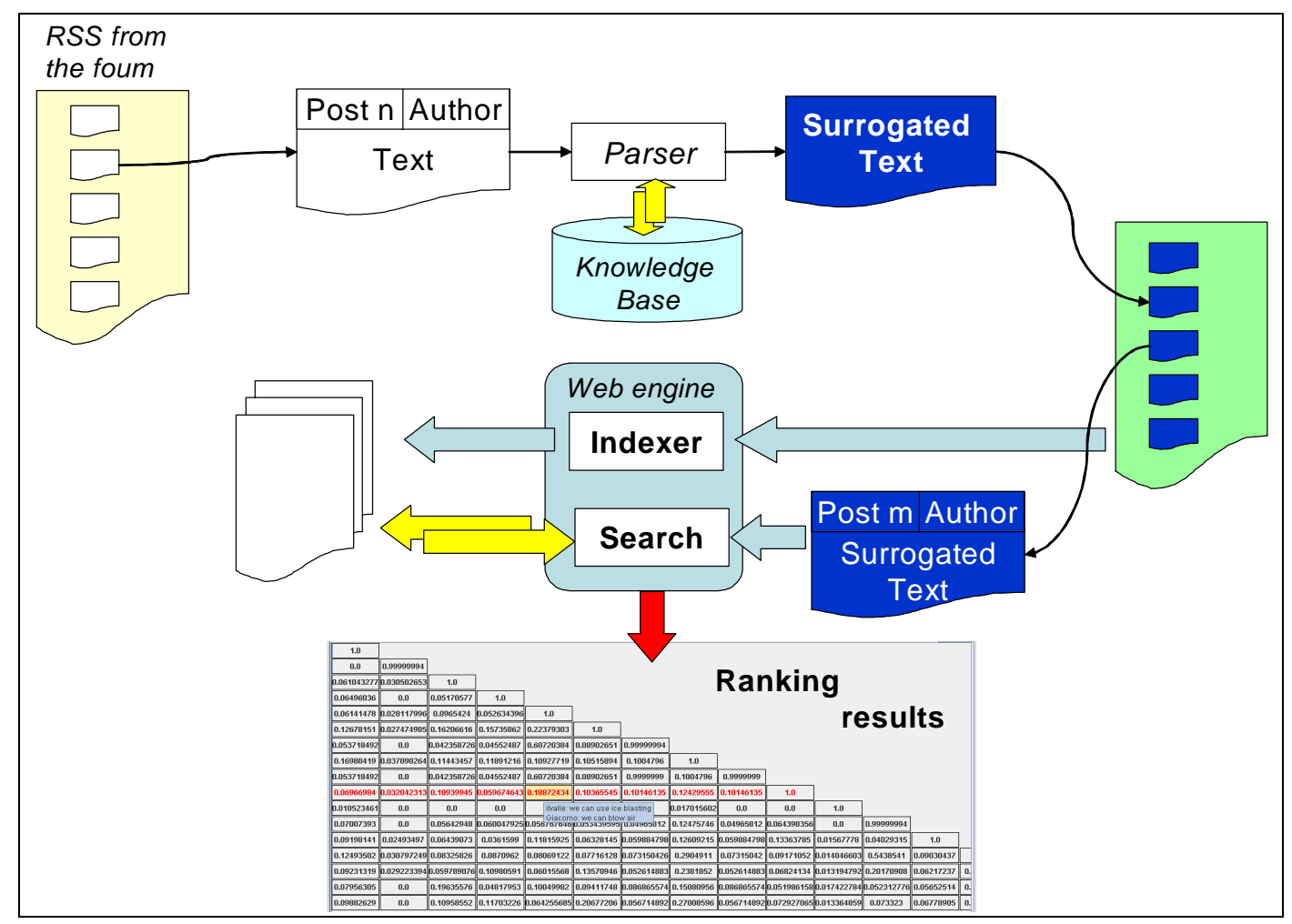

Figure 1: Software diagram

\section{Findings}

To test the efficiency of the system we performed a case study. The participants, remotely interacting through an advanced chat, were asked to suggest methods to remove dirtiness from a certain surface. Participants were invited to follow the rules of a standard brainstorming and to freely collaborate to reach the best solution. At the end of the session, an independent expert selected the "winning" proposal.

All contributions were then treated by the IPR tracking software that:

- numbered and ordered them on a time scale distributed among different lines corresponding to the users (Figure 2);

- indicated with coloured links the existing dependencies of each contribution from the precedent posts (different colours indicated different ranking values);

- $\quad$ performed analyses towards both preceding and following posts (Figure 3);

- finally, on the basis of the relationships found and according to a suitable algorithm, assigned to each participant a percentage indicating the degree of its contribution to the final solution. 


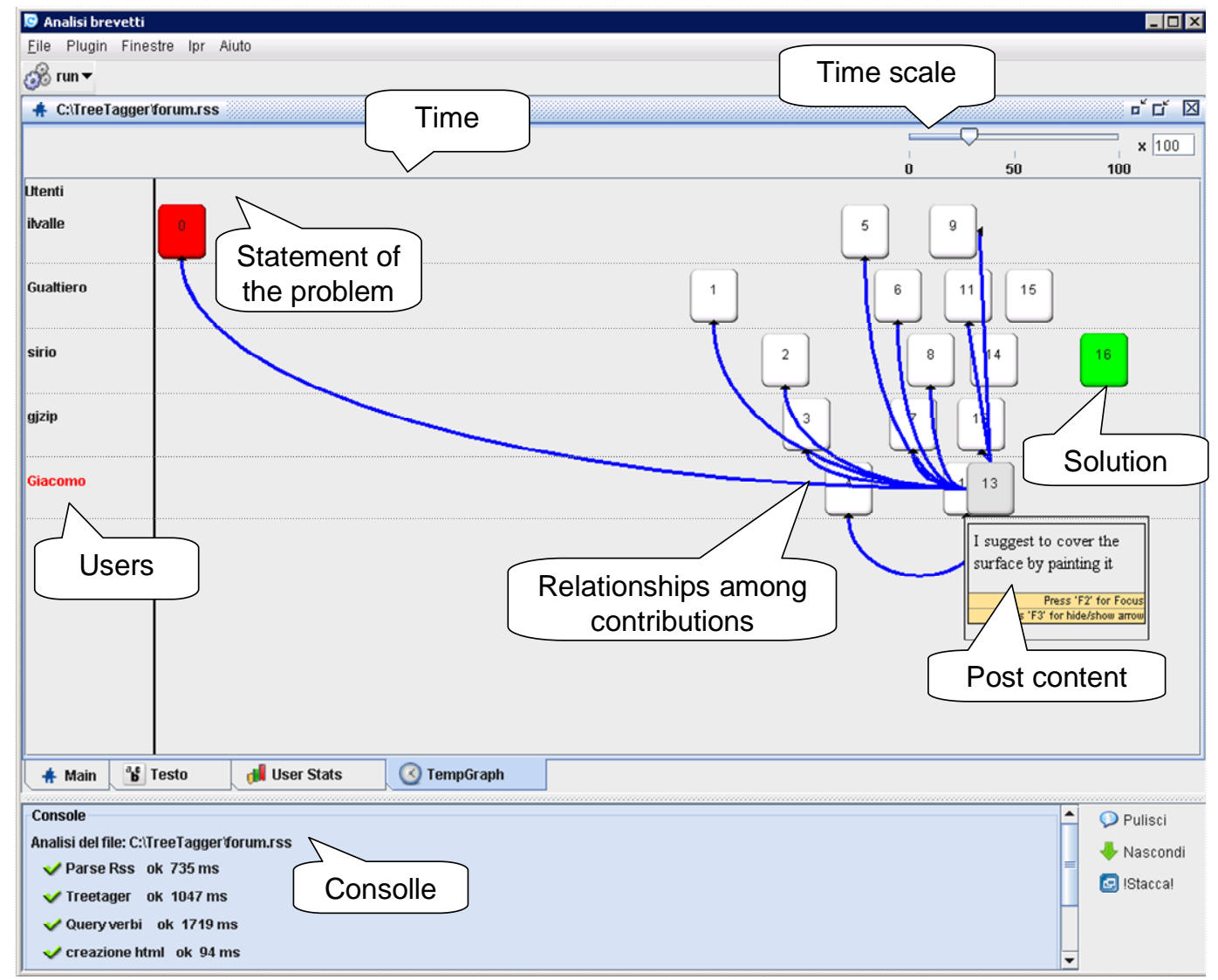

Figure 2: The IPR Tracking at work

Figure 2, 3 and 4 show the results of the processing. In Figure 2 and 3 all contributions, indicated by the numbered rectangles, are listed on a time scale and distributed among different lines corresponding to the six solvers. The software also indicates with coloured links the existing dependencies of each contribution from the precedent posts.

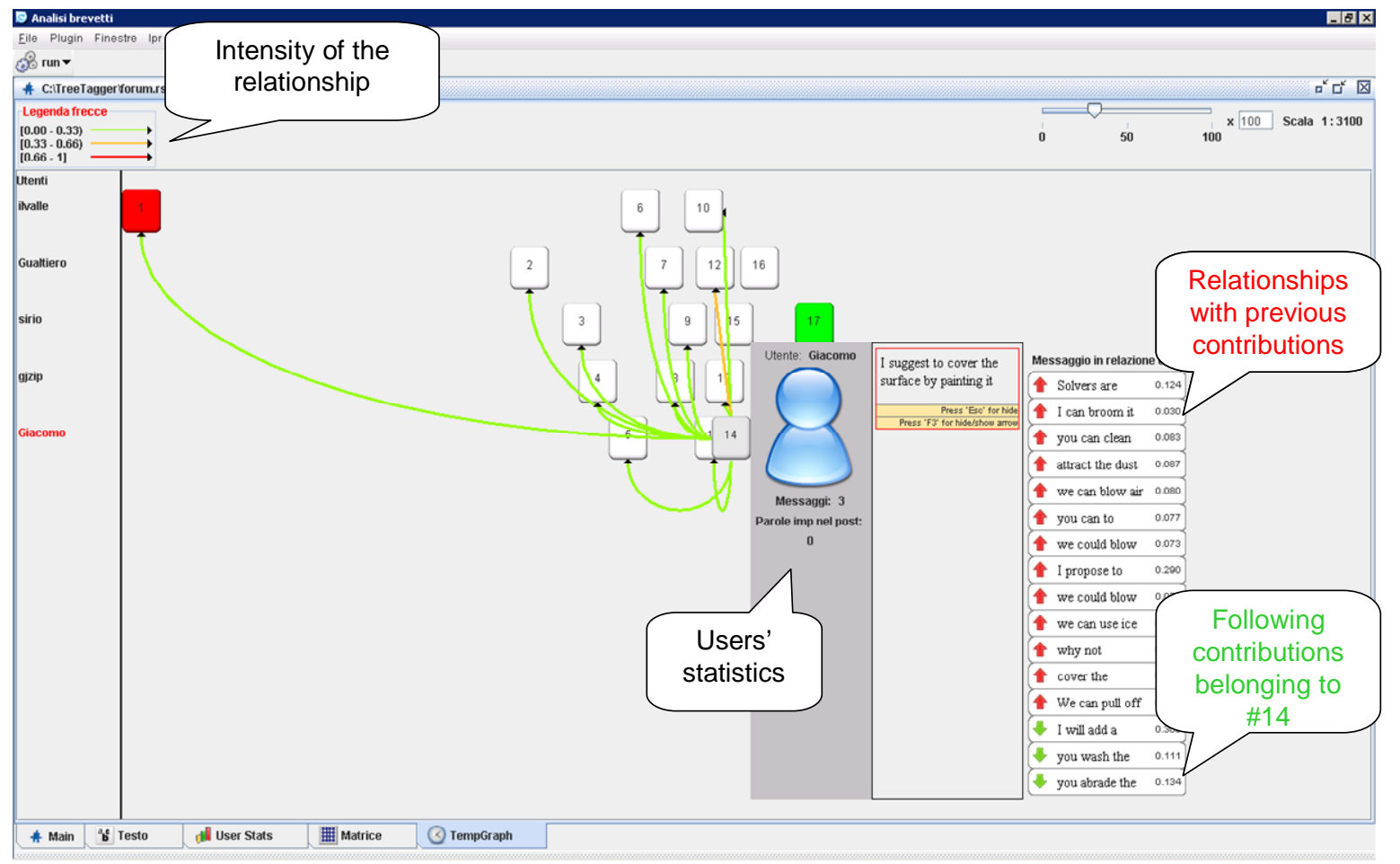

Figure 3: Dependencies and relationships among ideas 
For a link to exist, the value of the distance between statements should be below a stated threshold. Different colours are related to higher and higher value of the scalar products, hence to closer relationships (see Figure 3). The analysis can be performed towards both preceding and following posts.

Finally, the IPR tracking engine, on the basis of the relationships found and according to a suitable algorithm, assign to each participant a percentage indicating the degree of its contribution to the final solution (see Figure 3).

Before disclosing the software's result we asked the solvers to assess the relevance of each participant's contribution to the solution selected. The difference between the average of the human evaluations and the automatic estimate has been of order fifteen percent or less.

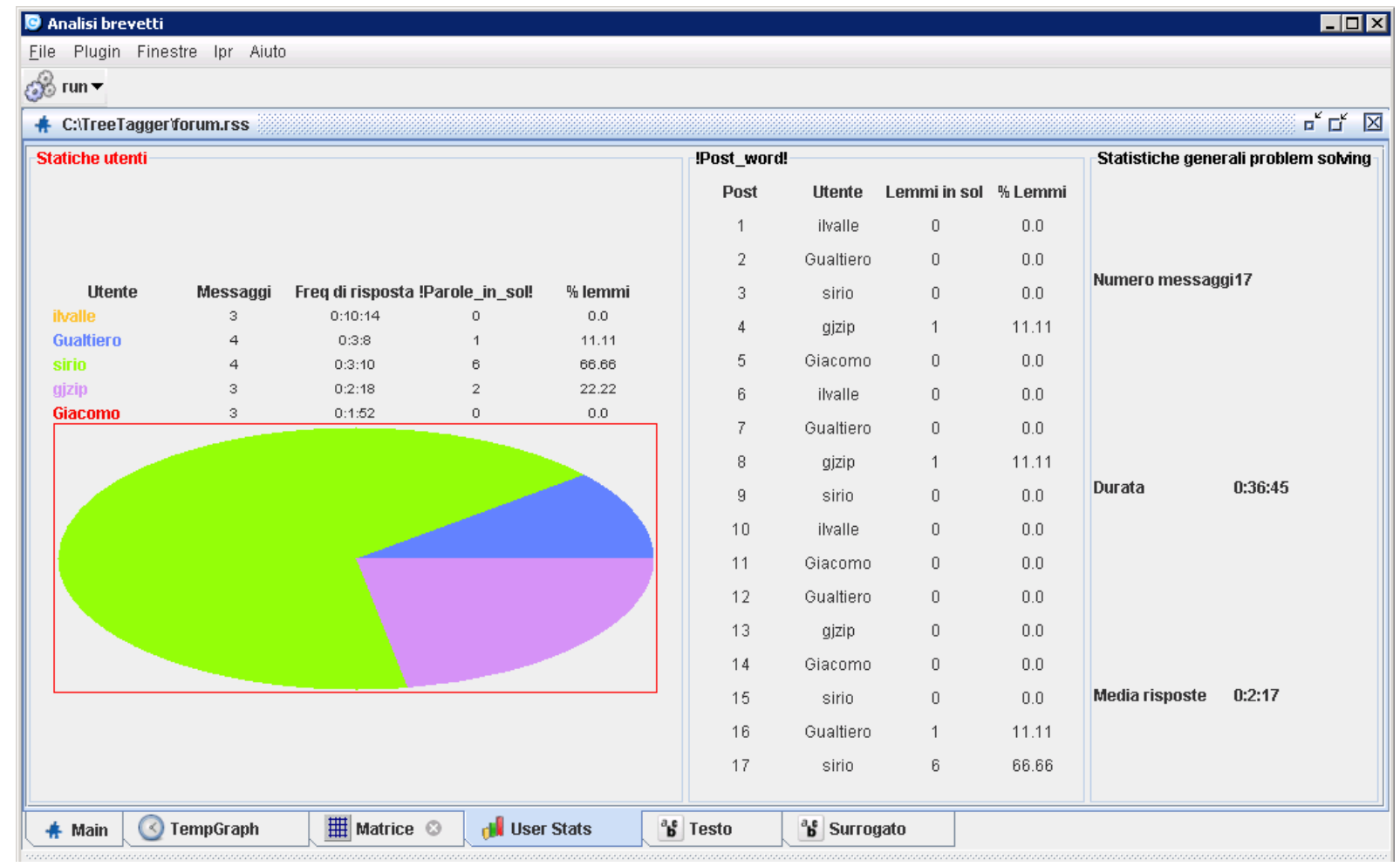

Figure 4: Users' statistics and additional statistical data

\section{Conclusion}

The management of collaborative environments is a very challenging task. The difficulties increase in large, heterogeneous and multidisciplinar groups of people accessing the service asynchronously. We presented here an IT platform powered by a web forum and an IPR tracking system that assists the users in collaborative crowdsourcing.

Participants feel free to express their ideas, are confident in a objective tracking system, knows that improving other interesting ideas can be the best way to achieve a common good result. Such environment boosts motivation, trust and participation. Moreover, since the existence of a public weighting system and protocol, participants can give their contributions to the co-creative effort being confident in a fair reward. Indeed, after the introduction of the IPR tracking protocol in the Leaning Lab, its activities have increased in both number and efficiency. 


\section{Acknowledgements}

The financial support of PRIME Network of Excellence (EU FP6th), Italian Ministry of Research FIRB-IRIS and PRIN2006 is kindly acknowledged.

\section{References}

ALIVE “Advanced Legal Issues in Virtual Enterprises” Project [IST-2000-25459]

Bifulco A., Santoro R., 2005, A conceptual framework for "professional virtual communities" Proceeding of the PRO-VE 2005, Valencia 26-28 September '05.

Borgo S., Carrara M., Garbacz P. and Vermaas P. E. (2008) A Formal Ontological Perspective on the Behaviors and Functions of Technical Artifacts, to appear in AIEDAM, special issue on "Developing and Using Engineering Ontologies".

Cascini G. (2003) System and Method for performing functional analyses making use of a plurality of inputs, Patent Application 02425149.8, European Patent Office, 14.3.2002, International Publication Number WO 03/077154 A2.

Cascini G. and Neri F. (2004) Multilanguage patents analysis and classification, TRIZ Future Conference, pp.199210.

Dong, A., Agogino, M. (1997) Text Analysis for Constructing Design Representation, Journal of artificial intelligence in Engineering vol 11(2).

Fantoni, Bifulco, Santoro (2006) Problem classes and collaborative methods: a new approach to innovation, ICE 2006

Kitamura Y. Kasai T. and Mizoguchi R. (2001) Ontology-based description of functional design knowledge and its use in a functional way server, Pacific Asian Conference on Intelligent Systems.

Lee Fleming, (2004) Perfecting Cross-pollination. Harvard Business review, September edition.

Pisano Gary P., Verganti R. (2008) Which Kind of Collaboration is Right for You? Harvard Business Review, December edition.

Leaning Lab. WWW page. http://www.leaninglab.org 